\title{
Gravitational Radiation from Gamma-Ray Burst Progenitors
}

\author{
Shiho Kobayashi and Peter Mészáros \\ Center for Gravitational Wave Physics, \\ Dept. of Astronomy $\& 3$ Astrophysics and Dept. of Physics, \\ Pennsylvania State University, 104 Davey Lab, University Park, PA 16802
}

\begin{abstract}
We study gravitational radiation from various proposed gamma-ray burst (GRB) progenitor models, in particular compact mergers and massive stellar collapses. These models have in common a high angular rotation rate, and the final stage involves a rotating black hole and accretion disk system. We consider the in-spiral, merger and ringing phases, and for massive collapses we consider the possible effects of asymmetric collapse and break-up, as well bar-mode instabilities in the disks. We calculate the strain and frequency of the gravitational waves expected from various progenitors, at distances based on occurrence rate estimates. Based on simplifying assumptions, we give estimates of the probability of detection of gravitational waves by the advanced LIGO system from the different GRB scenarios.
\end{abstract}

Subject headings: gravitational waves - binaries:close - black hole physics stars:neutron - gamma rays: bursts

\section{Introduction}

The recent discovery of long-lasting afterglows of gamma-ray bursts (GRBs) at longer wavelengths has revolutionized this field (e.g. van Paradijs, Kouveliotou \& Wijers 2000) and has greatly increased confidence in the relativistic blast wave model as an explanation for the afterglow electromagnetic signatures. As of now, upward of forty GRBs have been accurately localized, belonging to the class of so-called long bursts ( $\gamma$-ray durations upwards of $2 \mathrm{~s}$ ), and found to be predominantly in active star-forming regions. Although we lack direct evidence about the nature of the central engine producing the relativistic flow, it is nevertheless widely accepted that GRBs are the results of catastrophic events involving either compact stellar mergers or massive stellar collapses (for reviews, see Piran 2000; Mészáros 2002; Woosley 2001). Since the ratio of the total duration of the bursts to the variability timescales is large, 
GRB $\gamma$-rays must be produced by internal shocks or other dissipative events. A key feature of internal shocks is that the observed gamma-ray variability reflects the variability in the activity of the central engine (Kobayashi, Piran \& Sari 1997). Since variability timescales as short as a millisecond are observed, the engine must contain a compact object of less than a few solar masses, otherwise the light crossing time becomes larger than the variability timescales.

While the fastest variability timescale is comparable to the dynamical timescale of a millisecond, burst durations are usually very much longer, and the central engine must be active much longer than its dynamical time. This suggests that GRBs are powered by accretion disks and that the accretion timescales determine the durations. The observed energy in GRBs requires a massive $\left(\geq 0.1-1 M_{\odot}\right)$ disk. Such a massive disk can form from the fall-back of debris during the formation of the compact object itself, which ultimately is likely to be a newborn black hole. Several scenarios could lead to a black hole-massive accretion disk system. This includes the merger of double neutron star binaries (Eichler et al 1989; Ruffert et al. 1997), neutron star - black hole binaries (Paczynski 1991; Janka et al. 1999), black hole - white dwarf binaries (Fryer et al. 1999a), black hole - helium star binaries (Fryer \& Woosley 1998; Zhang \& Fryer 2001)

The present and foreseeable sensitivity of gravitational wave detectors is such that for likely sources, including GRB, the detections would be difficult, and for this reason, much effort has been devoted to the development of data analysis techniques that can reach deep into the detector noise. A coincidence between a gravitational wave signal and a gamma-ray signal would greatly enhance the statistical significance of the detection of the gravitational wave signal (Finn, Mohanty \& Romano, 1999; Kochanek \& Piran 1993). Therefore, it is of interest to study the gravitational wave emission from GRB associated with specific progenitors. Another reason for doing this is that, since the $\gamma$-rays and the afterglow are thought to be produced at very large distances $\left(\geq 10^{13} \mathrm{~cm}\right)$ from the central engine, we have only very indirect information about the nature of the latter. However, gravitational waves should be emitted from the immediate neighborhood of the GRB central engine itself, and their observation should give valuable information about its identity.

In the frequency band $\sim 10-1000 \mathrm{~Hz}$ relevant for the laser interferometer gravitational wave observatory (LIGO), and other detectors such as VIRGO, GEO600 and TAMA300 which are currently in operation, being developed or planned, the most promising sources of gravitational radiation are thought to be coalescing compact binaries (e.g. Phinney, 1991; Ruffert et al 1997, Janka et al 1999). Compact binary mergers may be responsible for short bursts (e.g. Woosley, 2001), but there is no observational supporting evidence for this as yet. On the other hand, those GRB which have been unambiguously localized 
and identified (all of them long bursts with $t_{\gamma} \gtrsim 10 \mathrm{~s}$ ) are likelier to be associated with massive stellar collapses (van Paradijs et al 2000). Numerical calculations of gravitational wave radiation from massive rotating stellar collapses have been done in the Newtonian approximation, for "collapsar" models of long GRB in 2D (e.g. Fryer et al. 1999a, McFadyen \& Woosley 1999), and in 3D for general cases not intended as models for GRB (Rampp et al 1998). These numerical estimates are not conclusive, as a number of effects (including general relativity, secular evolution, etc, see Rampp et al 1998) have been neglected, but they suggest that gravitational wave emission from massive collapses may be much less important than from compact binary mergers. On the other hand, recent semi-analytical estimates (Fryer, Holz \& Hughes 2002; Davies et al 2002; van Putten 2001) have indicated that instabilities in the collapsing core or in the accretion disk of a collapsar GRB could lead to significantly stronger gravitational wave signals than expected from the previous numerical estimates. It is therefore of interest to re-examine the gravitational wave signals expected from various specific GRB progenitors that have been recently discussed, and based on current astrophysical models, to consider the range of rates and strains expected in each case, for comparison with the LIGO sensitivity.

In $\S 2$ we review the mechanisms that lead to gravitational wave emission from GRB. In $\S 3$ we apply them to each of the GRB progenitor models and estimate the expected rates, strain and frequency of the gravitational waves. In $\S 4$, the detectability of the gravitational waves with the advanced LIGO system is discussed. We give conclusions in $\S 5$.

\section{Emission Mechanisms}

The process of binary coalescence as a gravitational wave source is in principle simpler to analyze than that of a massive stellar collapse, although they share some common features, especially in the later phases. The binary coalescence process can be divided into three phases: in-spiral, merger and ring-down (e.g., Flanagan \& Hughes 1998). (1) During the in-spiral phase, the gravitational radiation reaction time scale is much longer than the orbital period. As the binary loses energy by gravitational radiation, the masses gradually spiral in toward each other. (2) The merger begins when the orbital evolution is so rapid that adiabatic evolution is not a good approximation, or when the masses (if the radii are much larger than their gravitational radius, e.g. white dwarfs and Helium stars), come into contact with each other. Then the two masses go through a violent dynamical merger phase which leads to a black hole on a dynamical timescale, releasing a fraction of their rest mass energy in gravitational waves. However, a significant fraction of the stellar material retains too much angular momentum to cross the black hole horizon promptly. This creates a temporary disk 
of debris material around the $\mathrm{BH}$, whose accretion over times long compared to a dynamic time can power a GRB jet. In the case of a BH-WD or BH-He binary merger, the BH-disk phase is reached through a common-envelope phase in which the $\mathrm{BH}$ spirals in towards the center of the disrupted companion. Numerical simulations are accuracy-limited and strongly dependent on the assumptions and approximations made. A useful approach allowing semianalytical estimates is to roughly approximate the process as two masses, the $\mathrm{BH}$ and a mass comparable to the fraction of the companion mass which is inside the orbit the latter makes around the center of mass, spiraling towards each other. If the massive accretion disk which forms in the latter part of this process is unstable against self-gravity, perturbations in the disk itself can also lead to non-axisymmetric inhomogeneities whose mass is some fraction of the disk mass inside the corresponding radius, leading to gravitational radiation. (3) The black hole, right after it forms, is initially deformed, and in a ring-down phase radiates away the energy associated with these deformations as gravitational waves, until it settles into a Kerr geometry.

Collapsars, i.e. massive stellar collapses leading to a GRB, require a high core rotation rate, which may be easier to achieve if the star is in a binary system, although this is not necessary (e.g. Woosley, 2001). The high rotation rate is required to form a centrifugally supported disk around a central, possibly spinning black hole, to power a GRB jet. A high rotation rate, however, may be conducive to the development of bar or fragmentation instabilities in the collapsing core or/and in the massive disk around the central object (Nakamura \& Fukugita 1989; Bonnell \& Pringle 1995; van Putten 2001, 2002; Davies et al. 2002; Fryer, Holz \& Hughes 2002). The asymmetrically infalling matter also perturbs the black hole's geometry, which leads to rig-down gravitational radiation. The gravitational wave emission from collapsars can thus in principle be estimated in a similar way to what is done in binaries during the in-spiral, merger and ring-down phases, although with considerably larger uncertainties.

\subsection{In-spiral}

In-spiraling compact binaries can be described as two point particles with masses $m_{1}$ and $m_{2}$, whose orbital parameters evolve secularly due to gravitational radiation. The radiation carries away orbital binding energy, which leads to a faster orbiting and more compact system. Though the amplitude of the gravitational wave itself $h(t)$ increases as the system evolves, the frequency $f$ also rapidly increases. As the result, the energy spectrum is a 
decreasing function of $f$ (e.g., Misner, Thorne \& Wheeler 1973),

$$
\frac{d E}{d f}=\frac{(\pi G)^{2 / 3}}{3} \mathcal{M}^{5 / 3} f^{-1 / 3},
$$

where $\mathcal{M}=\left(m_{1} m_{2}\right)^{3 / 5}\left(m_{1}+m_{2}\right)^{-1 / 5}$ is the chirp mass. The characteristic gravitational wave amplitude is defined with the Fourier transform of $h(t)$ as $h_{c}(f)=f|\tilde{h}(f)|$, and equal to $\sim \sqrt{N} h$ where $N=f^{2}(d f / d t)^{-1}$ is the number of cycles radiated while the frequency changes by an amount of order $f$. The characteristic amplitude at a detector at a distance $d$ is given by a function of the energy spectrum.

$$
\begin{aligned}
h_{c} & =\frac{1}{\pi d} \sqrt{\frac{G}{10 c^{3}} \frac{d E}{d f}} \\
& \sim 1.4 \times 10^{-21}\left(\frac{d}{10 M p c}\right)^{-1}\left(\frac{\mathcal{M}}{M_{\odot}}\right)^{5 / 6}\left(\frac{f}{100 H z}\right)^{-1 / 6},
\end{aligned}
$$

This formula is obtained from an rms average of the amplitudes over different possible orientations of the source and interferometer. Since we used the standard definition of the signal-to-noise ratio defined by eq. (15) in section 4, our characteristic amplitude is smaller by a factor of $2 \sqrt{5}$ than eq. (5.1) in Flanagan \& Hughes (1998). For a given energy spectrum of gravitational waves and noise spectrum density, both formulae give the same signal-tonoise ratio.

\subsection{Merger}

Late in its evolution, a binary system will undergo a transition from an adiabatic inspiral induced by gravitational radiation damping to an unstable plunge induced by strong spacetime curvature (merger phase). For double neutron star binaries, the in-spiral signal contribution can be taken to end around the frequency (Kidder, Will \& Wiseman 1993; Lai \& Wiseman 1996),

$$
f_{i} \sim 1000\left(\frac{M}{2.8 M_{\odot}}\right)^{-1} \mathrm{~Hz},
$$

where the total mass $M=m_{1}+m_{2}$. However, if a white dwarf or a helium star consists of the binary, the masses collide with each other at a separation $l$ comparable to the sizes of masses. Since this happens well before the relativistic or tidal effect becomes important, the in-spiral gravitational signal ends at a much lower frequency,

$$
f_{i} \sim 0.1\left(\frac{M}{M_{\odot}}\right)^{1 / 2}\left(\frac{l}{10^{9} \mathrm{~cm}}\right)^{-3 / 2} \mathrm{~Hz} .
$$


The gravitational wave emission resulting from the coalescence of either double neutron star or black hole - neutron star binary systems is still poorly understood. The gravitational fields are quite strong and dynamical, which rules out a perturbative approach, and requires solving the full Einstein equations. Recently, numerical simulations of the coalescence of two equal mass black holes in off-axis collisions have been calculated and provide some guidance (Khanna et al. 1999). They find that about $1 \%$ of the total mass energy will emerge as gravitational waves during the final stages of the collision (ring-down phase). The radiation from the early merger stage of coalescence could be very much larger than the late stage ring-down radiation. Therefore, we assume that total energy radiated in the merger phase is

$$
E_{m}=\epsilon_{m}\left(\frac{4 \mu}{M}\right)^{2} M c^{2}
$$

where $\epsilon_{m}$ (of nominal value $5 \%$ ) is a parametrisation of the total mass energy radiated in the coalescence, and the reduced mass is $\mu=m_{1} m_{2} / M$. The reduction factor $(4 \mu / M)^{2}$ is unity for equal masses and gives the correct scaling law in the test particle limit $\mu \ll M$.

The frequency of gravitational radiation in the in-spiral phase is well-defined as a function of time, and increases monotonically. All the energy emitted in the in-spiral phase is at frequencies less than $f_{i}$. We assume that the spectrum of radiation in the merger phase is confined to the frequency regime $f>f_{i}$. As we discuss below, we define the end of the merger phase to occur when the waveform can be described by the $l=m=2$ quasi-normal mode signal of a Kerr black hole. The quasi-normal ringing frequency $f_{q}$ gives an approximate upper-bound for the frequencies carrying substantial power during the merger (Flanagan \& Hughes 1998).

$$
f_{q} \sim \frac{F(a) c^{3}}{2 \pi G M} \sim 32 F(a)\left(\frac{M}{M_{\odot}}\right)^{-1} \mathrm{kHz}
$$

where $F(a)=1-0.63(1-a)^{3 / 10}$ and $a$ is the dimensionless spin parameter of the black hole (Echeverria 1989). The simplest estimate of the energy spectrum is a flat spectrum with the following amplitude,

$$
\frac{d E}{d f}=\frac{E_{m}}{f_{q}-f_{i}}
$$

Using eq (2) and an approximation $f_{q}-f_{i} \sim f_{q}$, the characteristic gravitational wave amplitude is given by

$$
h_{c} \sim 2.7 \times 10^{-22} F(a)^{-1 / 2}\left(\frac{\epsilon_{m}}{0.05}\right)^{1 / 2}\left(\frac{4 \mu}{M}\right)\left(\frac{d}{10 M p c}\right)^{-1}\left(\frac{M}{M_{\odot}}\right) .
$$

Here the $M, \mu$ and $\epsilon_{m}$ are those appropriate to the end of the merger phase. 
If dynamical instabilities develop in the rotating core or in the rotating massive disk during the merger phase, the deformed core/disk could radiate strong gravitational waves in a narrow frequency band. The deformation may be considered, in its simplest form, as either two blobs or a bar. Using in either case a formula appropriate for a rotating bar (e.g. Fryer, Holz \& Hughes 2002), we can estimate the amplitude of the corresponding gravitational wave emission. Considering a bar of mass $m$ and length $2 r$ which rotates with angular frequency $\omega$, the mean strain is given by

$$
h=\sqrt{\frac{32}{45}} \frac{G}{c^{4}} \frac{m r^{2} \omega^{2}}{d} .
$$

Assuming $\omega^{2}=G m^{\prime} / r^{3}$, the characteristic amplitude $h_{c} \equiv \sqrt{N} h$ is

$$
h_{c} \sim 1.9 \times 10^{-21}\left(\frac{N}{10}\right)^{1 / 2}\left(\frac{m}{M_{\odot}}\right)\left(\frac{m^{\prime}}{M_{\odot}}\right)\left(\frac{d}{10 M p c}\right)^{-1}\left(\frac{r}{10^{6} \mathrm{~cm}}\right)^{-1} .
$$

where we assumed that the waves remain coherent for $N=10$ cycles. When we discuss the instability of a rotating core, $m$ and $m^{\prime}$ are assumed to be equal and their cannot exceed the mass of the core, while when we discuss the instability of an accretion disk rotating around a black hole, $m$ and $m^{\prime}$ are assumed to be the masses of the accretion disk and the black hole, respectively.

\subsection{Ring-down}

A deformed black hole should undergo damped vibrations which emit gravitational radiation. The most slowly damped mode, which has spherical harmonic indices $l=m=2$, will dominate over other quasi-normal modes at late times. Since this mode may also be preferentially excited in the presence of binary masses or fragmentation of a massive disk, we here focus attention on the $l=m=2$ mode.

The spectrum is peaked at $f_{q}$ with a width given by the inverse of the damping time $\Delta f \sim \tau^{-1}=\pi f_{q} / Q(a)$ where $Q(a)=2(1-a)^{-9 / 20}$ (Echeverria 1989),

$$
\frac{d E}{d f} \sim \frac{E_{r} f^{2}}{4 \pi^{4} f_{q}^{2} \tau^{3}}\left[\frac{1}{\left[\left(f-f_{q}\right)^{2}+(2 \pi \tau)^{-2}\right]^{2}}+\frac{1}{\left[\left(f+f_{q}\right)^{2}+(2 \pi \tau)^{-2}\right]^{2}}\right],
$$

where $E_{r}=\epsilon_{r}(4 \mu / M)^{2} M c^{2}$ is the energy radiated during the ring-down phase, and we assume $\epsilon_{r}=0.01$ as a nominal parameter henceforth. The characteristic gravitational wave amplitude at $f=f_{q}$ is given by

$$
h_{c} \sim 2.0 \times 10^{-21}\left(\frac{\epsilon_{r}}{0.01}\right)^{1 / 2}\left(\frac{Q / F}{14}\right)^{1 / 2}\left(\frac{d}{10 M p c}\right)^{-1}\left(\frac{\mu}{M_{\odot}}\right) .
$$


The value of the spin $a$ of the final black hole depends on the initial parameters of the system, and this dependence is not well understood at present. Since the black hole may typically have spun up to near maximal rotation by a massive accretion disk (Thorne 1974), we adopt $a=0.98$ which is the value assumed by Flanagan \& Hughes (1998).

\section{Progenitors}

In recent years the black hole accretion disk model for GRBs has received much attention (Fryer et al. 1999b; Popham, Woosley \& Fryer 1999; Mészáros 2000, 2002; Narayan, Piran \& Kumar 2001). Progenitors likely to lead to this accretion system include binary mergers and collapsars: double neutron stars (DNS), black hole - neutron star (BH-NS), black hole - white dwarf (BH-WD), black hole - helium star (BH-He), and fast-rotating massive stellar collapses. If the viscosity parameter of the disks has a standard value of $\alpha=0.1$, DNS and BH-NS mergers can explain short GRBs with durations under a second, but they are unlikely to produce long GRBs with durations of tens or hundred of seconds. On the other hand, BH-WD and BH - He mergers and collapsars might produce long GRBs.

Recently Fryer et al. (1999b) and Belczynski, Bulik \& Rudak (2002) have estimated the formation rate of these progenitors by using population synthesis methods. The results of Fryer et al. (1999b) are summarized in Table 1, where the standard values of the formation rates and the uncertainty ranges are listed. Assuming the galaxy density $n_{\text {glx }}=0.02 \mathrm{Mpc}^{-3}$, we can estimate the distance inside which an event is expected to happen within in a year from the formation rates $R$.

$$
d \sim 230\left(\frac{R}{\mathrm{Myr}^{-1} \text { galaxy }^{-1}}\right)^{-1 / 3}\left(\frac{n_{\text {glx }}}{0.02 \mathrm{Mpc}^{-3}}\right)^{-1 / 3} \mathrm{Mpc} .
$$

Table 1

\begin{tabular}{lcccc}
\hline & \multicolumn{2}{c}{$\begin{array}{c}\text { Formation Rate } \\
{\left[\mathrm{Myr}^{-1} \text { galaxy }^{-1}\right]}\end{array}$} & \multicolumn{2}{c}{$\begin{array}{c}\text { Distance } \\
{[\mathrm{Mpc}]}\end{array}$} \\
& Standard & Range & Standard & Range \\
\hline DNS & 1.2 & $0.01-80$ & 220 & $53-1100$ \\
BH-NS(a) & 2.6 & $0.001-50$ & 170 & $62-2300$ \\
$\quad$ (b) & 0.55 & $0.001-50$ & 280 & $62-2300$ \\
BH-WD & 0.15 & $0.0001-1$ & 430 & $230-4900$ \\
BH-He & 14 & $0.1-50$ & 95 & $62-490$ \\
Collapsar & 630 & $10-1000$ & 27 & $23-110$ \\
\hline
\end{tabular}




\subsection{Double Neutron Stars}

Stars more massive than $\sim 8 M_{\odot}$ are thought to collapse to form a neutron star remnant and a core-collapse supernovae, while stars more massive than $\sim 25 M_{\odot}$ are thought to result in a black hole remnant. The standard scenario to form close double neutron star (DNS) binaries begins with two massive stars with masses between $\sim 8 M_{\odot}$ and $\sim 25 M_{\odot}$. The more massive (primary) star in the binary evolves off the main sequence, and forms a neutron star accompanied by a supernova explosion. If the system remains bound after the supernova explosion of the primary, it results in a binary composed of a neutron star and a massive main sequence star. When the secondary, in turn, evolves off the main sequence and expands, the neutron star enters the hydrogen envelope of the secondary and begins to spiral toward the secondary's helium core. The orbital energy released ejects the hydrogen envelope, forming a neutron star - helium star binary. After the explosion of the helium star as a supernova, there remains a DNS binary.

However, recent calculations of neutron star accretion reveal that, during this common envelope phase, the neutron star can accrete over $1 M_{\odot}$, and collapse to form a black hole. Thus, the standard scenario for DNS binaries may in fact form BH-NS binaries (Chevalier 1996; Bethe \& Brown 1998; Fryer et al. 1999b). An alternative scenario (Brown 1995) is that the initial binary system consists of two massive stars with nearly equal mass (within $5 \%$ difference). The secondary evolves off the main sequence before the explosion of the primary as a neutron star. The two stars then enter a common envelope phase and form double helium star binary. After the hydrogen envelope is ejected the helium stars explode and collapse to neutron stars.

The solid line in figure 1 gives the characteristic amplitude of gravitational waves from a DNS binary $\left(m_{1}=m_{2}=1.4 M_{\odot}\right)$ in the in-spiral phase at $220 \mathrm{Mpc}$. This distance corresponds to the radius within which a merger event is expected in a year, according to the rates in table 1 . The shaded region shows the uncertainty range corresponding to the formation rate uncertainty. Around $f_{i} \sim 1000 \mathrm{~Hz}$, the orbital evolution becomes so rapid that adiabatic evolution is not a good approximation any more. Then, the neutron stars begin to merge, they quickly form an object too large to be supported by nuclear and degeneracy pressure. A black hole forms on a dynamical time-scale, but a significant amount of mass $\sim 0.03-0.3 M_{\odot}$ will have, at first, too much angular momentum to fall into the hole promptly (Fryer et al. 1999b). The resultant accretion disk and/or the spin of the black hole (via the Blandford-Znajek [1977] mechanism) is responsible in this model for the relativistic jet which powers the electromagnetic signal of the GRB (Mészáros \& Rees 1997).

The amplitudes of the gravitational wave signals associated with the merging (dasheddotted) and ring-down phases (solid spike) of the black hole are also shown in Figure 1. The 
frequency of the $\mathrm{BH}$ quasi-normal mode is $\sim 9.3 \mathrm{kHz}$. In addition, a bar-mode instability might develop in the early stage of the merger, or a similar instability may arise intermittently in the inner disk. To illustrate the maximum signal levels that could arise from such instabilities, in equation (11) we used arbitrary but plausible parameters $m=m^{\prime}=2.8 M_{\odot}$, $r=4 G \mathrm{~m} / \mathrm{c}^{2}$ and assuming that the waves remain coherent for $N=10$ cycles, we have plotted in the same figure the characteristic strain (circle), with an error bar due to the uncertainty in the formation rate.

\subsection{Black Hole - Neutron Star}

As discussed above, BH-NS binaries can form from neutron star binaries in which the neutron star of the primary undergoes too much accretion during the common envelope phase and collapses to a black hole. This formation scenario ( $a$ in Table 1) produces a binary consisting of a neutron star of $m_{2} \sim 1.4 M_{\odot}$ and a low-mass black hole of $m_{1} \sim 3 M_{\odot}$ (Fryer et al. 1999b).

The more standard formation scenario for BH-NS system (scenario $b$ in Table 1 ) begins with two massive stars in which the primary have a mass greater than $\sim 25 M_{\odot}$. The primary evolves off the main sequence, and continues to evolve. When it forms a black hole, the system consists of a black hole and a massive star. This system evolves through a common envelope phase as the secondary star expands. During this common envelope phase, the black hole spirals into the massive secondary and ejects the hydrogen envelope. The supernova explosion of the secondary results in the formation of a binary consisting of a neutron star and a higher mass black hole $m_{1} \sim 12 M_{\odot}$ (see Fig 17 in Fryer et al. 1999b).

We assume that the gravitational wave signal from the in-spiral binary ends around the frequency given by eq. (4) and hence this signal shuts off nominally at $f_{i} \sim 640 \mathrm{~Hz}$ (scenario a) or $f_{i} \sim 210 \mathrm{~Hz}$ (scenario $b$ ). When the BH-NS binary merges, the neutron star is tidally disrupted. Some of the material accretes onto the BH directly, while the remainder forms an accretion disk of $0.3-0.7 M_{\odot}$ (Janka et al. 1999). The electromagnetic GRB signal from BH-NS is similar to that in DNS binaries, and of comparable but somewhat larger energy (Mészáros \& Rees 1997). The gravitational wave signals are also comparable, the differences being associated with the $\mathrm{BH}$ mass (or possibly its spin rate, related to the total accretion history). We show the amplitudes of the gravitational waves for the two BH-NS scenarios in figure 2. The quasi-normal ring-down mode frequencies are $\sim 6 \mathrm{kHz}(a)$ and $\sim 2 \mathrm{kHz}(b)$. If a bar instability develops in the massive accretion disk, the maximum range of signals expected from this are calculated from equation (11) with optimal but plausible parameters $m=0.5 M_{\odot}$ and $r=6 G m^{\prime} / c^{2}$, rotating around a black hole of $m^{\prime}=4 M_{\odot}(a)$ or $=13 M_{\odot}$ 
(b), and assuming that the waves remain coherent for $N=10$ cycles. These are shown as circles in figure 2 .

\subsection{Black Hole - White Dwarf}

A scenario for binaries consisting of a black hole and a white dwarf (BH-WD) begins with main sequence stellar systems having an extreme mass ratio, with primary mass $\geq 25 M_{\odot}$ and secondary mass $\leq 8 M_{\odot}$. After the primary collapses to form a black hole, one has a binary consisting of a black hole $\sim 3-15 M_{\odot}$ and a main-sequence star (Fryer et al. 1999a). As the secondary star expands off the main sequence, a common envelope phase can occur, which results in a decrease of the orbital separation. The system eventually evolves into a binary of a black hole and a high mass white dwarf of mass $\geq 0.9 M_{\odot}$ (Fryer et al. 1999a). Since white dwarf radii $\sim 10^{9} \mathrm{~cm}$ are much larger than those of neutron stars, the gravitational wave signal from the in-spiral phase shuts off at a low frequency $f_{i} \sim 0.4\left(M / 10 M_{\odot}\right)^{1 / 2}\left(r / 10^{9} \mathrm{~cm}\right)^{-3 / 2} \mathrm{~Hz}$. This is well below the seismic noise cutoff $\sim 10 \mathrm{~Hz}$.

As the BH-WD binary undergoes merger, the BH spirals inward through the envelope of the white dwarf, in the process of which the white dwarf is tidally disrupted and most of its matter goes into an accretion disk around the black hole. Gravitational radiation is expected during this process from the varying quadrupole moment of the $\mathrm{BH}$ and the inner portions of the WD core or other inhomogeneities arising during the disruption. This can be described in a simplified manner as the gravitational radiation of a $\mathrm{BH}$ and one or more discrete time-variable mass concentrations as they approach and start to merge with each other. In figure 3, we give the characteristic maximum gravitational wave amplitude from the coalescence of a $\mathrm{BH}$ of $m_{1}=10 M_{\odot}$ and a white dwarf after the spiral-in phase (with mass values motivated by Fryer et al 1999a), during the merger and disruption phase. The fraction of the WD mass concentration used in equation (9) is $m_{2}=\alpha M_{\odot}$, where we take $\alpha \lesssim 10^{-1}$ as a rough upper limit of the mass fraction of the WD core or blob, including any infall, which lies inside a radius $6 G m_{1} / c^{2}$ of the $\mathrm{BH}$ at the time of close approach to the $\mathrm{BH}$ when the dynamical merger process starts. Bar instabilities may also form in a disk resulting from the tidal destruction of the WD, and we have plotted the gravitational wave amplitude from a bar with $m=\beta M_{\odot}$ (with $\beta \sim 10^{-1}$ ) and length $r=6 \mathrm{Gm}^{\prime} / \mathrm{c}^{2}$ rotating coherently for $N=10$ cycles around a black hole of $m^{\prime}=10 M_{\odot}$, to represent the maximal radiation from bar-unstable massive disk. Also plotted is the final BH ring-down phase signal, which is calculated from equation (13) with $\mu$ estimated with the same masses as the final merger signal. 


\subsection{Black Hole - Helium Star}

A helium star (BH-He) merger model begins with a close binary system of two massive stars, each greater than $8 M_{\odot}$. The more massive star (primary) evolves off the main sequence, and forms a neutron star in a supernova explosion. When the secondary, in turn, evolves off the main sequence and expands, the neutron star enters its companion's hydrogen envelope. If the in-spiraling neutron star does not have sufficient orbital energy to eject the hydrogen envelope, it moves on into the helium core. The neutron star quickly accretes enough material to become a black hole. The angular momentum of the black hole - helium core binary is injected into the helium core, forming a massive disk of mass $\sim 4 M_{\odot}$ around a spinning black hole of $\sim 3 M_{\odot}$, with a disk radius equal to a fraction of the initial helium core $\sim 10^{9}-10^{10}$ cm (Fryer \& Woosley 1998).

A He star is of comparable or somewhat larger outer radius than a WD and hence gravitational waves from the pre-contact in-spiral phase again lie below the seismic noise cut-off threshold. As in the BH-WD case, gravitational waves in the LIGO sensitivity range can arise after the $\mathrm{BH}$ penetrates the envelope of the He star and circles its way inwards, due to the varying quadrupole moment of the $\mathrm{BH}$ and the stellar core or inhomogeneities arising during the tidal destruction of the star, from bar instabilities in the deformed massive tidal disruption disk, and from the final ring-down of the BH. In order to estimate the maximum characteristic amplitude in the merging and ring-down phases, we use eqs (9) and (13) with $m_{1}=3 M_{\odot}$ and $m_{2}=4 \alpha M_{\odot}$ (with mass values motivated by Fryer \& Woosley 1998), where we took $\alpha \sim 10^{-1}$. The results are shown in figure 4. The maximum amplitude of the gravitational waves from a bar with mass $m=4 \beta M_{\odot}$ (where $\beta \sim 10^{-1}$ ) and length $r=6 \mathrm{Gm}^{\prime} / \mathrm{c}^{2}$ rotating coherently for $N=10$ cycles around a black hole with $m^{\prime}=3 M_{\odot}$ is also plotted.

\subsection{Collapsar}

If the progenitor mass exceeds $\sim 40 M_{\odot}$, the collapse of the iron core does not produce a successful outgoing shock but instead leads to the prompt formation of a black hole of $\sim 2-3 M_{\odot}$. For a GRB, it is required that the progenitor, or at least its core, be fast rotating (MacFadyen \& Woosley 1999; Fryer 1999). This scenario of prompt collapse to a $\mathrm{BH}$ is dubbed a type I collapsar. As the inner stellar layers fall into the newborn black hole, and form an accretion disk, the energy from the massive disk and/or the rotation of the black hole itself powers a GRB jet in the same way as in the previous scenarios. For

progenitor masses between $\sim 25 M_{\odot}$ and $\sim 40 M_{\odot}$ (e.g. Fryer 1999) the collapse of the iron core leads to a different scenario, since the initial core mass infall is not sufficient to make 
a BH promptly, but leads to a temporary NS and an initial outward-moving shock. After a delay of minutes to hours, the fall-back of stellar material that was initially moving outwards, but which fails to achieve escape velocity, finally drives the central object above the maximal NS mass limit $\sim 3 M_{\odot}$ (Rhoads \& Ruffini 1974), and a BH is formed, leading to a GRB. This is called the type II collapsar scenario. Although the accretion time-scales of type II collapsars may be too long for the average long bursts of $\sim 10-30$ sec, type II collapsars are probably as likely or somewhat more frequent events than Type I, because they involve a more densely populated portion of the stellar mass function. Assuming an initial mass function of $f d M \propto M^{-2.7} d M$, the ratio of the number of progenitor stars of both types is $N\left(25 M_{\odot}-40_{\odot}\right) / N\left(>40 M_{\odot}\right) \sim 1.2$.

Collapsars, even if arising from single stars, have in principle the potential to emit a substantial amount of their energy in gravitational waves, since they are fast-rotating and non-axisymmetries in the collapse are to expected. So far, the results of numerical calculations of massive rotating stellar collapses have not confirmed this. However, numerical calculations of collapsars models to date have been in the Newtonian approximation and in 2D (i.e. axi-symmetric, Fryer et al. 1999a, McFadyen \& Woosley 1999), while Newtonian 3D collapse calculations were not intended particularly as GRB models (Rampp et al 1998). Although these suggest that gravitational wave emission from massive collapses may be much less important than from compact binary mergers, it may be argued that these results are not conclusive, since a number of effects (including general relativity, secular evolution, etc, see Rampp et al 1998) have been neglected. If the rotating collapsing core fragments to produce two or more compact objects (Nakamura \& Fukugita 1989), their coalescence could emit strong gravitational waves. Also, although the mass of the accretion disk in such systems depends on the viscosity assumed, the disk mass is as high as $\sim 1 M_{\odot}$ in the low-viscosity models of MacFadyen \& Woosley (1999). In this case, the self-gravity of the disk will become important, and gravitational instabilities (e.g., spiral arm or bar formation) can develop and radiate gravitational waves (Davies et al 2002; Fryer et al 2002; van Putten 2002). The black hole will also suffer deformations from the non-uniform, non-axisymmetric accreting material, leading to ring-down.

The characteristic gravitational wave amplitudes are calculated in a similar way as before, under the assumption that the core collapse leads to asymmetrical blobs which undergo a merger leading to a $\mathrm{BH}$, which then undergoes a ring-down phase. The parameters chosen are plausible but arbitrarily chosen to represent the maximum level of signals that could be expected. These are shown in figure 5, where we assumed $m_{1}=m_{2}=\alpha M_{\odot}$ with $\alpha \lesssim 1$ (representing blobs which become the $\mathrm{BH}$ ) and we assumed that the merging phase starts when the blobs are separated by $r \sim 10^{7} \mathrm{~cm}$. A rough estimate on the maximal emission from an unstable accretion disk is given by the gravitational wave amplitudes from bars of 
$m=\beta M_{\odot}, m^{\prime}=3 M_{\odot}$ and $r=6 G m^{\prime} / c^{2}$ where $\beta \lesssim 1$. We have calculated these amplitudes for the distances derived from the occurrence rates of Type I collapsars derived by Fryer et al. (1999b). Type II rates were not computed, but if we take into account the Type II as well, using the mass function ratio in the next to last paragraph, the amplitude of gravitational waves from collapsars should be stronger than what is shown in figure 5, which corresponds to Type I only.

\section{Detectability}

A complete set of theoretical waveform templates will be available for the in-spiral and ring-down phases of compact binary mergers such as $\mathrm{BH}-\mathrm{BH}$, and presumably also for $\mathrm{BH}$ NS, NS-NS). These are directly applicable to the first two GRB scenarios, and may be of some limited use in other scenarios, e.g. if core collapse leads to break-up into very dense (NS-like) blobs. When templates can be used, one can employ the matched filtering technique (e.g. Thorne 1987) to optimize the search for gravitational wave signals in the observational data stream. The signal-to-noise ratio $(\mathrm{S} / \mathrm{N}) \rho$ is given by

$$
\rho^{2}=4 \int_{0}^{\infty} \frac{|\tilde{h}(f)|^{2}}{S_{h}(f)} d f
$$

where $S_{h}(f)$ is the noise power spectral density of the detector. The gravitational wave signal is detectable if the $\mathrm{S} / \mathrm{N}$ exceeds a threshold $\rho_{t h}$, as a rough rule of thumb taken as $\rho_{t h} \sim 5$.

$N S-N S$ and $B H-N S$ - The matched filtering technique can in general be applied only to the in-spiral phases of NS-NS and BH-NS binaries. Unfortunately, the ring-down frequencies of the quasi-normal modes of stellar mass black holes are too high to be optimal for the advanced LIGO. Furthermore, the in-spiral signal from BH-WD and BH-He binaries ends below the seismic cutoff frequency $\sim 10 \mathrm{~Hz}$. For the nearest NS-NS binary in-spiral which happens in a year, the $\mathrm{S} / \mathrm{N}$ is

$$
\rho_{N S-N S, \text { insp }} \sim 7.4(1.5,30 .)\left(\mathcal{M} / 1.2 M_{\odot}\right)^{5 / 6}\left(R / 1.2 \mathrm{Myr}^{-1} \text { galaxy }^{-1}\right)^{1 / 3}
$$

where the numbers in parenthesis give the range of the $\mathrm{S} / \mathrm{N}$ ratio due to the uncertainty in the formation rate $R$ listed in Table 1 , and $\mathcal{M}=\left(m_{1} m_{2}\right)^{3 / 5}\left(m_{1}+m_{2}\right)^{-1 / 5}$ is the chirp mass. For the nearest BH-NS binary in a year in scenario a and $\mathrm{b}$, the $\mathrm{S} / \mathrm{N}$ values and their uncertainties are

$$
\rho_{B H-N S, \text { ins }}= \begin{cases}13(0.9,35 .)\left(\mathcal{M} / 1.8 M_{\odot}\right)^{5 / 6}\left(R / 2.6 \mathrm{Myr}^{-1} \text { galaxy }^{-1}\right)^{1 / 3}, & \text { scenario (a) } \\ 12(1.5,54 .)\left(\mathcal{M} / 3.2 M_{\odot}\right)^{5 / 6}\left(R / 0.55 \mathrm{Myr}^{-1} \text { galaxy }^{-1}\right)^{1 / 3}, & \text { scenario (b) }\end{cases}
$$


The advanced LIGO system could therefore detect gravitational waves from these NS-NS and BH-NS scenarios.

Collapsars - For collapsars, as well as for BH-WD and BH-He star binaries, the gravitational wave frequencies in the merger phase (if the core breaks up into blobs) come into a suitable range for detection by the advanced LIGO. Although the waveform is unknown, the signals may nonetheless be detectable using the cross-correlation of the outputs of two LIGO detectors. The two LIGO detectors are coaligned, but widely separated. If we set the arrival time of the GRB signal as the origin of time at each detector by using the sky position of the GRB or the afterglow, we can correct for the physical separation so that the two detectors can be considered as "coincident" (i.e. having identical locations and arm orientations). The outputs of the two detectors around the onset of the GRB are

$$
s_{1}(t)=n_{1}(t)+h_{1}(t), \quad s_{2}(t)=n_{2}(t)+h_{2}(t),
$$

where $h_{1}(t)$ and $h_{2}(t)$ are the gravitational wave strains in the two detectors, and $n_{1}(t)$ and $n_{2}(t)$ denote the noise components intrinsic to the two detectors. Since we assume that the two detectors are coincident and coaligned, the gravitational strains are essentially identical, $h(t) \equiv h_{1}(t)=h_{2}(t)$. Given the detector outputs, we can define the weighted cross-correlation signal $X_{\text {on }}$ as

$$
X_{o n}=\left(s_{1}, s_{2}\right)=\int_{-T / 2}^{T / 2} d t \int_{-T / 2}^{T / 2} d t^{\prime} s_{1}(t) s_{2}\left(t^{\prime}\right) Q\left(t-t^{\prime}\right),
$$

where $Q$ is a filter function. We can also write this equation in the frequency domain.

$$
X_{o n} \sim \int_{-\infty}^{\infty} d f \int_{-\infty}^{\infty} d f^{\prime} \delta_{T}\left(f-f^{\prime}\right) \tilde{s}_{1}^{*}(f) \tilde{s}_{2}\left(f^{\prime}\right) \tilde{Q}\left(f^{\prime}\right),
$$

where $\tilde{s}_{1}, \tilde{s}_{2}$ and $\tilde{Q}$ are the Fourier transforms of $s_{1}(t), s_{2}\left(t^{\prime}\right)$ and $Q\left(t-t^{\prime}\right) . \quad \delta_{T}(f)=$ $\sin (\pi f T) / \pi f$ is the finite-time approximation to the Dirac delta function. If we knew the signal $h(t)$, we could construct a $Q$ that maximizes the $\mathrm{S} / \mathrm{N}$ as $\tilde{Q}(f)=|\tilde{h}(f)| / S_{1}(|f|) S_{2}(|f|)$. $S_{1}(f)$ and $S_{2}(f)$ are the power spectral densities of the noises of the two detectors. Finn et al. (1999) suggested to adopt this filter with $|\tilde{h}(f)|^{2}$ assumed to be unity in the detector band if a detailed knowledge of $h(t)$ is lacking. Therefore, assuming for simplicity that the power spectral densities are identical, $S(f) \equiv S_{1}(f)=S_{2}(f)$ in the two detectors, we adopt $\tilde{Q}(f)=\lambda / S^{2}(|f|)$ where $\lambda$ is a normalization constant.

The expected value of the cross-correlation signal (averaged over the source population) is

$$
\left\langle X_{o n}\right\rangle \sim \lambda \int_{-\infty}^{\infty} \frac{\left\langle h_{c}^{2}(f)\right\rangle}{f^{2} S^{2}(|f|)} d f .
$$


Using data segments not associated with GRBs (off-source), we can also evaluate the fluctuation of the cross-correlation of the noise,

$$
\sigma_{o f f}^{2}=\overline{\left(n_{1}, n_{2}\right)^{2}} \sim \frac{\lambda^{2} T}{4} \int_{-\infty}^{\infty} \frac{d f}{S^{2}(|f|)} .
$$

The factor $T$ on the right hand side arises from evaluating $\delta_{T}(0)$. We assume that the duration of gravitational wave bursts are shorter than $T=10 \mathrm{sec}$. In the previous section, we assumed that the merger phase starts at $\sim 200 \mathrm{~Hz}$ (collapsars), or at $f_{i} \sim 0.4 \mathrm{~Hz}(\mathrm{BH}-$ WD and BH-He binaries). During $T=10 \mathrm{sec}$, the system can rotate multiple times, and it is plausible to assume that a significant fraction of the energy in the system is emitted during this time. If the energy spectrum of the gravitational waves $d E / d f$ is flat, as we have assumed, most of gravitational wave energy is emitted in the high frequency band $\sim f_{q}$. The emission timescale might be comparable to the damping time of the quasi-normal mode $\tau$ (Flanagan \& Hughes 1998). Although $\tau$ is much shorter than $10 \mathrm{sec}$, the lag between a GRB signal and the gravitational wave signal are model-dependent and rather uncertain. We assume a conservative estimate of $T=10 \mathrm{sec}$.

We define the $\mathrm{S} / \mathrm{N}$ of the gravitational wave signal in the merger phase as $\bar{X}_{\text {on }} / \sigma_{\text {off }}$. For the nearest collapsar which occurs in a year, the $\mathrm{S} / \mathrm{N}$ ratio calculated numerically from equations $(21)(22)$ is

$$
\rho_{\text {Coll,merg }} \sim 3\left(\frac{\epsilon_{m}}{0.05}\right)\left(\frac{F(a)}{0.8}\right)^{-1}\left(\frac{T}{10 \mathrm{sec}}\right)^{-1 / 2}\left(\frac{\mu}{0.5 M_{\odot}}\right)^{2}\left(\frac{R}{630 \mathrm{Myr}^{-1} \text { galaxy }^{-1}}\right)^{2 / 3}
$$

where the uncertainty range in $\rho$ from the uncertainty range in the rate $R$ is $0.2-3.8$ (from Table 1), the blob masses enter through the reduced mass $\mu=m_{1} m_{2} /\left(m_{1}+m_{2}\right)$, and we assumed that the blob merger starts at $r=10^{7} \mathrm{~cm}$ (which determines the range of frequency overlap between the signal and noise curves of Figure 5), and 5\% of the gravitational energy of the merging blobs is radiated away. For collapsars, this estimate is highly dependent on the assumed radius at which the merging phase of the blobs starts (and of course on the assumption that break-up into blobs is prevalent). If we assume that the merger starts at $r \sim 3 \times 10^{7} \mathrm{~cm}$, instead of $10^{7} \mathrm{~cm}$ as assumed for the previous estimate above, the gravitational wave signal shown as a dot-dashed line and shaded band in Figure 5 moves further left, and completely overlaps the most sensitive band $\sim 50-500 \mathrm{~Hz}$ of the detector. The $\mathrm{S} / \mathrm{N}$ is then $\rho_{\text {Coll,merg }} \sim 4.5(0.3-6.2)$, with other parameters scaling as in equation (23). The same equation (23) with a factor 4.5 in front is applicable also to BHWD mergers (with $\mu \sim 0.1 M_{\odot}, R \sim 0.15 \mathrm{Myr}^{-1}$ galaxy $^{-1}$, see $\S 3.3$ ) and BH-He mergers (with $\mu \sim 0.4 M_{\odot}, R \sim 14 \mathrm{Myr}^{-1}$ galaxy $^{-1}$, see $\left.\S 3.4\right)$, with the signal frequency range also spanning the LIGO sensitivity curve. This leads to $\rho_{B H-W D \text {,merg }} \sim 7 \times 10^{-4}\left(5 \times 10^{-6}-2 \times 10^{-3}\right)$, and $\rho_{B H-H e, \text { merg }} \sim 0.2\left(7 \times 10^{-3}-0.4\right)$, respectively. Thus, for BH-He and BH-WD binaries the 
$\mathrm{S} / \mathrm{N}$ ratio is too low. However, for collapsars using the above parameters, the $\mathrm{S} / \mathrm{N}$ is near the threshold of detectability. Therefore, gravitational waves from the collapsar scenario of GRB assuming break-up of the core into blobs could be marginally detectable through the cross-correlation technique.

$\mathrm{BH}-\mathrm{WD}$ and $\mathrm{BH}-\mathrm{He}$ - The BH-WD and BH-He binary scenarios of GRB are the least favorable for detection, because the value of the gravitational wave cross-correlations between two detectors is very small. In this case, one might still obtain some information on a possible association between GRBs and gravitational wave bursts, or upper limits on the corresponding strains, by collecting many samples. If the gravitational wave bursts are associated with GRBs, the correlated output of the two detectors will be different during the times when GRBs occur (on-source) than during other times not associated with a GRBs (off-source). A statistically significant difference between on- and off-source cross-correlation measures would support the association. This difference can be measured using Student's $t$-test (Finn et al. 1999). If $\sqrt{N_{o n}}\left\langle X_{o n}\right\rangle / \sigma_{o f f}$ is greater than a critical value (say 2.58 for $99 \%$ significance), where $N_{o n}$ is the number of the on-source events, we can conclude that we have found evidence for the association. The number of BH-He/WD binary events needed to detect the association with $99 \%$ confidence is

$$
N_{B H-H e / W D} \sim\left(\frac{\left\langle X_{o n}\right\rangle / \sigma_{o f f}}{2.58}\right)^{-2}
$$

If we assume that GRBs within a Hubble radius $(\sim 3000 \mathrm{Mpc})$ are detectable and that the $\mathrm{BH}-\mathrm{He} / \mathrm{WD}$ event rate is uniform, the typical distance to the event is $\sim 2000 \mathrm{Mpc}$. The $\mathrm{S} / \mathrm{N}$ of the gravitational wave signal from a binary at $2000 \mathrm{Mpc}$ is $\rho_{B H-W D \text {,merg }} \sim$ $3 \times 10^{-5}$ and $\rho_{B H-H e, m e r g} \sim 4 \times 10^{-4}$. The required number of events is $N_{B H-W D} \sim$ $7 \times 10^{9}\left(\epsilon_{m} / 0.05\right)^{-2}(F / 0.8)^{2}(T / 10 \mathrm{sec})\left(\mu / 0.1 M_{\odot}\right)^{-4}(d / 2000 M p c)^{4}$ and $N_{B H-H e} \sim 4 \times 10^{7}$ $\left(\epsilon_{m} / 0.05\right)^{-2}(F / 0.8)^{2}(T / 10 \mathrm{sec})\left(\mu / 0.4 M_{\odot}\right)^{-4}(d / 2000 M p c)^{4}$. We might be able to select only nearby events by using redshift observations of the afterglows, if available. When we analyze the nearest $n$ events in a year, the typical distance $d$ is proportional to $n^{1 / 3}$. Since the number of events needed to detect the association is $\propto d^{4} \propto n^{4 / 3}$, the number of years it takes to collect samples is $\propto n^{1 / 3}$. When we collect only the nearest event in a year $n=1$, the number of events needed to detect the association is $N_{B H-W D} \sim 10^{7}\left(\epsilon_{m} / 0.05\right)^{-2}$ $(F / 0.8)^{2}(T / 10 \mathrm{sec})\left(\mu / 0.1 M_{\odot}\right)^{-4}\left(R / 0.15 \mathrm{Myr}^{-1} \mathrm{gal}^{-1}\right)^{-4 / 3}$ and $N_{B H-H e} \sim 200\left(\epsilon_{m} / 0.05\right)^{-2}$ $(F / 0.8)^{2}(T / 10 \mathrm{sec})\left(\mu / 0.4 M_{\odot}\right)^{-4}\left(R / 14 \mathrm{Myr}^{-1} \mathrm{gal}^{-1}\right)^{-4 / 3}$, or equivalently, it takes $10^{7}$ years and 200 years to collect the samples. The ranges of $N_{B H-W D}$ and $N_{B H-H e}$ due to the uncertainty of the formation rates are $10^{6}-10^{11}$ and $37-10^{5}$, respectively. Thus, it is unlikely that one can detect an association between GRBs and gravitational waves from BH-WD or BH-He binaries. However, with a relatively small number sample, it is still possible to set a tight upper limit on the amplitude of the gravitational waves. If we have 
$N_{B H-W D / H e}=30$ events of BH-WD or BH-He binaries at any distances and we detect no signal of gravitational waves, we can give an upper limit on the mean amplitude of the events $h_{c}<7.2 \times 10^{-23}\left(N_{B H-W D / H e} / 30\right)^{-1 / 4}(T / 10 \mathrm{sec})^{1 / 4}$.

\section{Conclusions}

We have estimated the strains of gravitational waves from some of the most widely discussed current GRB progenitor stellar systems. If some fraction of GRBs are produced by double neutron star or neutron star - black hole mergers, the gravitational wave chirp signal of the in-spiral phase should be detectable by the advanced LIGO within one year, associated with the GRB electromagnetic signal. We have also estimated the signals from the black hole ring-down phase, as well as the possible contribution of a bar configuration from gravitational instability in the accretion disk following tidal disruption or infall in GRB scenarios. Among the other progenitor scenarios, the signals from black hole - Helium star and black hole - white dwarf merger GRB progenitors are the least likely to be detectable, due to the low estimates obtained for the maximum non-axisymmetrical perturbations. For another possible type of GRB progenitor, the massive rotating stellar collapses or collapsars, the non-axisymmetrical perturbations may be stronger, and the estimated formation rates are much higher than for other progenitors, with typical distances correspondingly much nearer to Earth. This type of progenitor is of special interest, since it has so far received the most observational support from GRB afterglow electromagnetic observations. For collapsars, in the absence of detailed numerical 3D calculations specifically aimed at GRB progenitors, we have roughly estimated the strongest signals that might be expected in the case of bar instabilities occurring in the accretion disk around the resulting black hole, and in the maximal version of the recently proposed fragmentation scenario of the infalling core. Although the waveforms of the gravitational waves produced in the break-up, merger and/or bar instability phase of collapsars are not known, a cross-correlation technique can be used making use of two co-aligned detectors. Under these assumptions, collapsar GRB models would be expected to be marginally detectable as gravitational wave sources by the advanced LIGO within one year of observations.

Collapsars are suspected to be responsible for many of the long GRBs, with $\gamma$-ray durations $t_{\gamma} \gtrsim 2 \mathrm{~s}$ (van Paradijs et al 2000), which have an occurrence rate $\sim 2 \mathrm{Gpc}^{-3} \mathrm{yr}^{-1}$,

or $\sim 0.1 \mathrm{Myr}^{-1}$ galaxy $^{-1}$. The short GRBs, with $t_{\gamma} \lesssim 2 \mathrm{~s}$, have an occurrence rate which is somewhat uncertain, but there are indications that they might be ten times more frequent than long ones (Piran 2002). If compact binary mergers, such as double neutron stars and black hole - neutron star mergers lead to GRB, these should be of the short variety (Popham 
et al 1999). Such mergers are relatively simpler systems, which are estimated with greater confidence to be strong gravitational wave emitters. The actual occurrence rates would be higher by a factor of $2 / \theta^{2}$ if the $\gamma$-ray emission of GRBs is not isotropic but is beamed within an opening angle $\theta$. Current afterglow observations (available so far mostly for long bursts of $t_{\gamma} \gtrsim 10 \mathrm{~s}$ ) indicate that long GRBs are beamed with opening angles of a few to a few ten degrees. There is so far no evidence for beaming in short GRB. However, binary mergers also have natural channels along their rotational axis (although not as constraining as the walls of the extended stellar cores in collapsars), and they may therefore also be beamed into some as yet undetermined opening angle (which is thought to be wider than in long bursts). The formation rates listed in Table 1 are consistent with the observed GRB rates when considering the beaming factors, if these are assumed similar for all progenitors. As a consequence of the beaming, GRBs may not be observable in $\gamma$-rays from all progenitor events, due to misalignment between the observer and jet axes. In such cases, the so-called "orphan" afterglows in other electromagnetic wavebands (X-ray, optical etc), and/or other GRB related transient events such as X-ray flashes (e.g. van Paradijs et al 2000) may be detectable. Such orphan electromagnetic burst events would also be associated with the (much less collimated) gravitational wave signals, which vary only by a factor $\sim 2$ between the equator and the pole, the latter being along the rotation or GRB jet axis.

We would like to thank L. Sam Finn, Ben Owen, Patrick Sutton and Ian Jones for useful discussions and valuable comments. We acknowledge support through the Center for Gravitational Wave Physics, which is funded by NSF under cooperative agreement PHY 01-14375, and through NSF AST0098416 and NASA NAG5-9192. 


\section{References}

Bethe,H. \& Brown,G.E. 1998, ApJ, 506, 780.

Blandford,R.D. \& Znajek,R.L. 1977, MNRAS, 179, 433.

Brown,G.E. 1995, ApJ, 440, 270.

Bonnell, I.A. \& Pringle, J.E. 1995, MNRAS, 273, L12.

Belczynski,K., Bulik,T. \& Rudak,B. 2002, ApJ, 571, 394.

Chevalier,R.A. 1996, ApJ, 459, 322.

Davies, M.B. et al. 2002, submitted to ApJ. Lett., astro-ph/0204358.

Eichler, D. et al. 1989, Nature, 340, 126.

Echeverria, F. 1989, Phys. Rev. D, 40, 3194.

Finn,L.S., Mohanty,S.D. \& Romano, J.D. 1999, Phys. Rev. D, 60, 121101.

Flanagan,E.E. \& Hughes, S.A. 1998, Phys. Rev. D, 57, 4535.

Fryer, C.L. 1999, ApJ, 522, 413.

Fryer, C.L. et al. 1999a, ApJ, 520, 650.

Fryer, C.L. et al. 1999b, ApJ, 526, 152.

Fryer, C.L. \& Woosley, S.E. 1998, ApJ, 502, L9.

Fryer, C.L., Holz, D.E. \& Hughes,S.A. 2002, ApJ, 565, 430.

Janka,H.T. et al. 1999, ApJ, 527, L39.

Khanna, G. et al. 1999, Phys. Rev. Lett., 83, 3581.

Kidder,L.E., Will, C.M. \& Wiseman,A.G. 1993, Phys. Rev. D, 47, 3281.

Kobayashi,S., Piran,T. \& Sari, R 1997, ApJ, 490, 92.

Kochanek,C. \& Piran, T. 1993, ApJ, 417, L17.

Lai,D. \& Wisenman, A.G. 1996, Phys. Rev. D, 54, 3958.

MacFadyen,A.I. \& Woosley,S.E. 1999, ApJ, 524, 262.

Mészáros, P. and Rees, M.J. 1997, ApJ, 482, L29

Mészáros, P. 2000, Nucl. Phys. B, 80, 63.

Mészáros, P. 2002, Annu. Rev. Astron. Astrophys., 40,137.

Misner,C.W., Thorne,K.S. \& Wheeler,J.A., Gravitation (Freeman, San Francisco 1973).

Nakamura,T. \& Fukugita,M. 1989, ApJ, 337, 466.

Narayan,R., Piran,T. \& Kumar,P. 2001, ApJ, 557, 949.

Paczynski,B. 1991, Acta Astronomica, 41, 257.

Phinney,E.S. 1991, ApJ, 380, L17.

Piran, T. 2000, Phys. Rep., 333, 529.

Piran, T. 2002, Review talk given at GR16, preprint gr-qc/0205045.

Popham,R., Woosley,S.E. \& Fryer,C. 1999, ApJ, 518,356.

Rampp,M., Muller,E. \& Ruffert,M. 1998, A\&A, 332, 969.

Rhoads,C.E. \& Ruffini,R 1974, Phys. Rev. Lett., 32, 324.

Ruffert, M. et al. 1997, A\&A, 319, 122. 
Thorne,K.S. 1974, ApJ, 191, 507.

Thorne,K.S., in 300 Years of Gravitation, edited by S.W. Hawking and W. Israel (Cambridge University Press, Cambridge, 1987) p 330-458.

van Putten, M.H.P.M. 2001 , ApJ Lett., 562, L51.

van Putten, M.H.P.M. 2002 , appear in ApJ Lett, astro-ph/0207242.

van Paradijs,J, Kouveliotou,C \& Wijers,R.A.M.J, 2000, Annu.Rev.Astron.Astrophys, 38, 379

Woosley,S. 2001, Proc. Rome Symp., Eds. E. Costa, F. Frontera, and J. Hjorth (Springer:Berlin) p. 257 Zhang,W. \& Fryer,C.L. 2001, ApJ, 550, 357. 


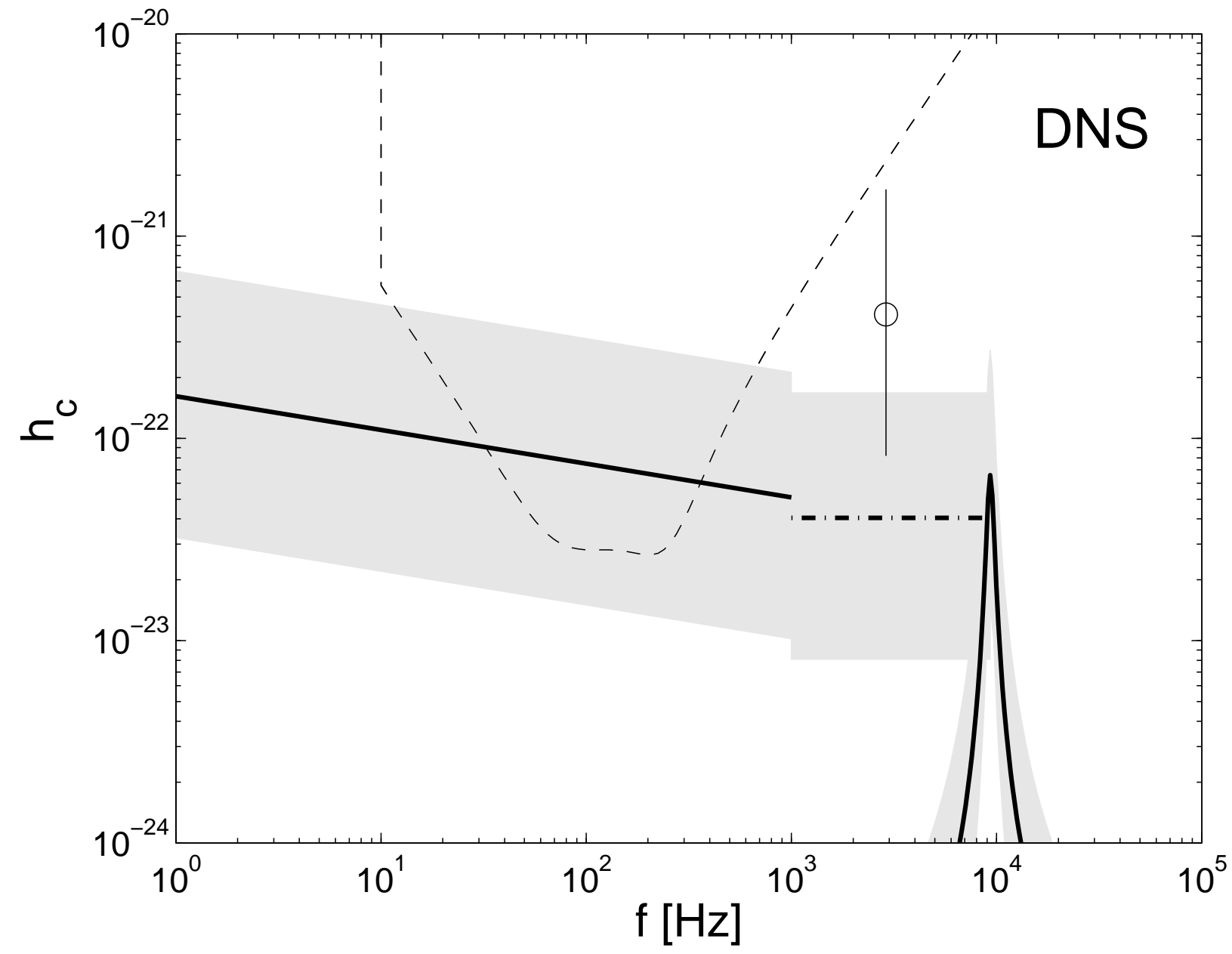

Fig. 1.- Double neutron stars : in-spiral (solid line), merger (dashed dotted line), bar (circle), ring-down(solid spike), see discussion in §3.1. Also shown is the advanced LIGO noise curve $\sqrt{f S_{h}(f)}$ (dashed curve). The shaded region and the vertical line reflect the uncertainty of the formation rate $R$ in Table 1 . 


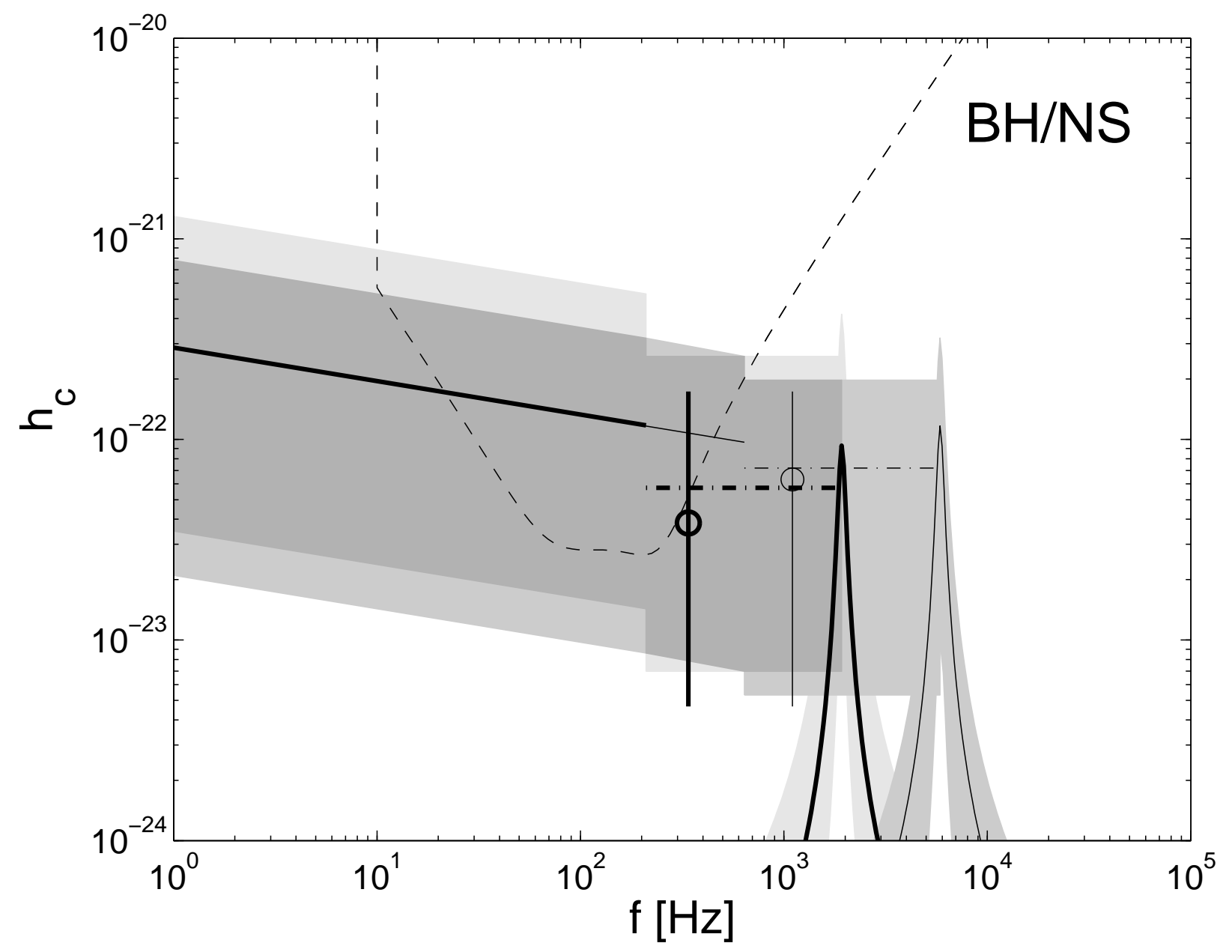

Fig. 2.- BH-NS, scenario a (thin lines and curve) and scenario b (thick lines and curve), for the parameters of §3.2: in-spiral (solid line), merger (dashed dotted line), bar (circle) and ring-down(solid spike). 


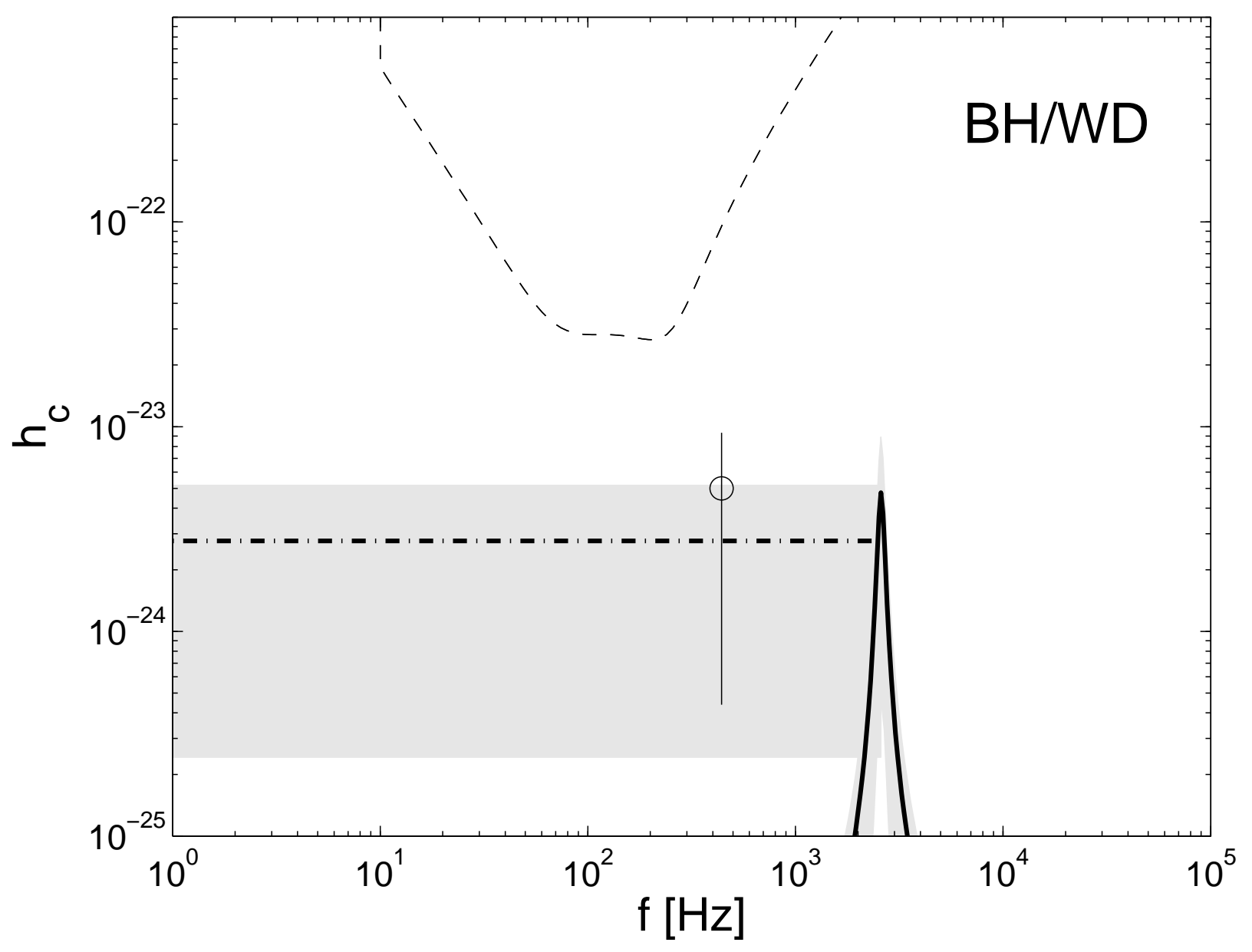

Fig. 3.- BH-WD: merger phase (dashed dotted line), bar (circle) and ring-down(solid spike), for the parameters and assumption discussed in $§ 3.3$. 


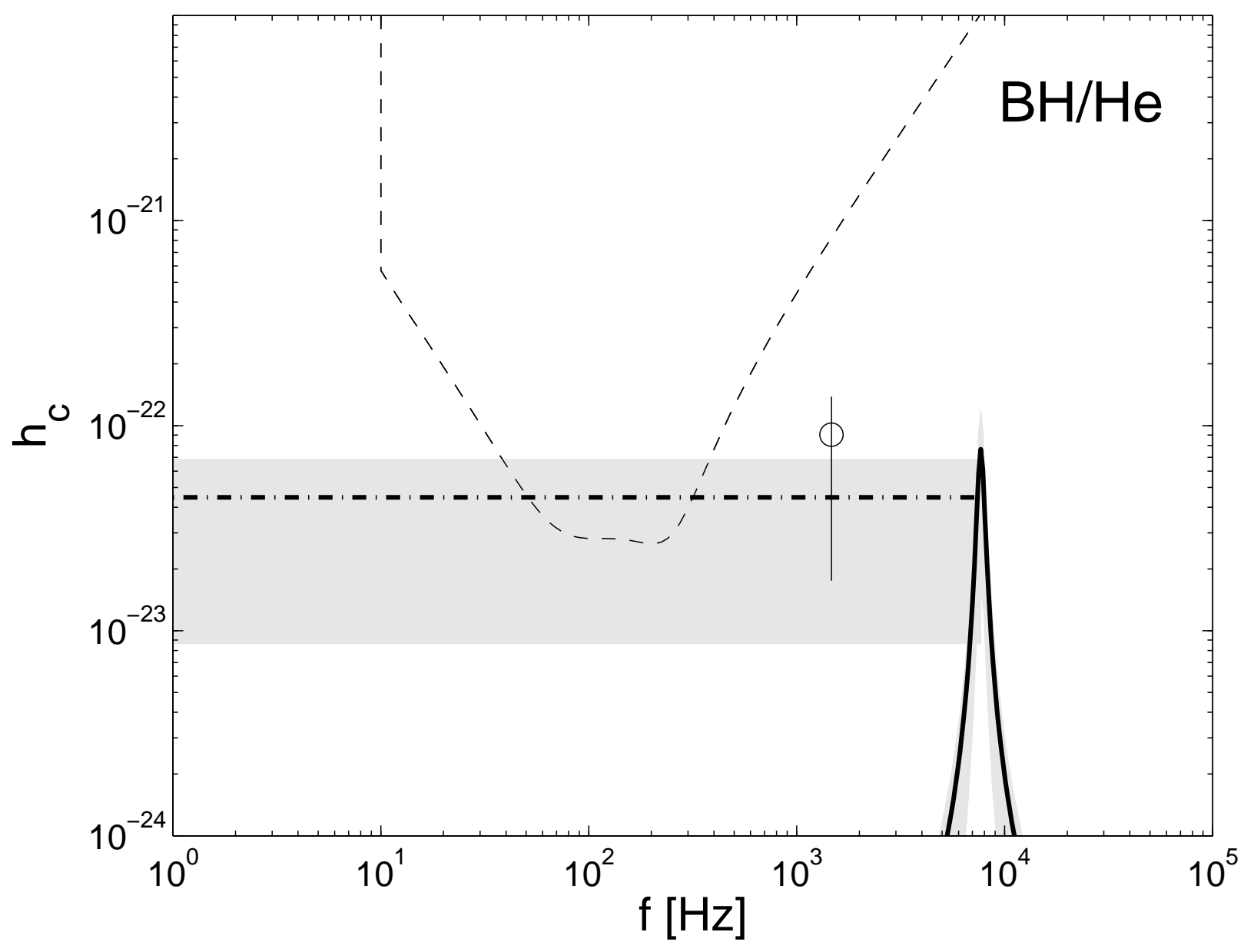

Fig. 4.- BH-He star: merger phase (dashed dotted line), bar (circle), and ring-down(solid spike), as discussed in $\S 3.4$. 


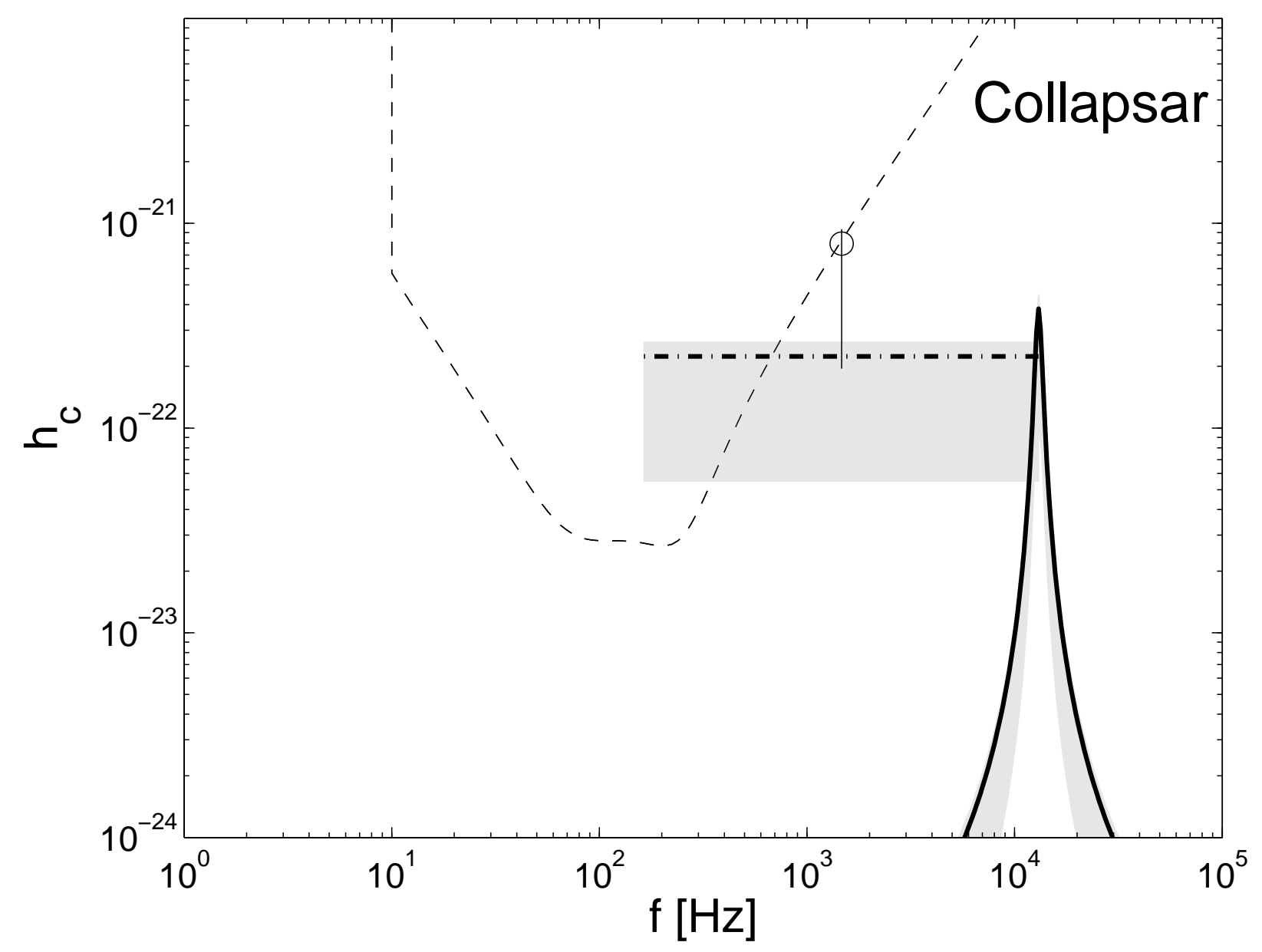

Fig. 5.- Collapsar: blob merger (dashed dotted line), bar (circle) and ring-down(solid spike), for the parameters discussed in $§ 3.5$. 